Introduction/Background* Borderline ovarian tumors (BOT) are low malignant potential lesions with a good prognosis that represent around $15 \%$ of all epithelial tumors of the ovary. In addition to that, $30 \%$ of patients with

BOT are less than 40 years old which makes the preservation of fertility a key point in management.

Molecular studies in ovarian cancer have shown a correlation between the genetic profile of the tumor and the patient's prognosis

Methodology We report the case of a 25 -year-old patient, G0P0A0, diagnosed with a mucinous borderline right ovarian tumor back in 2005. In 2009, during a routine control ultrasound, another ovarian cyst was identified on the right side. She was operated again of a cystectomy and peritoneal staging and histopathology confirmed the mucinous nature of the tumor. FIGO stage was IA in both cases. The two blocs of tumors underwent a full exome sequencing technique in order to identify possible key mutations.

Result(s)* Both tumors had variations of EGFR, FGFR3, BRCA1, STK11, NTKR1 and PIK3CA genes. However, the first tumor also had a KRAS mutation that wasn't found in the second lesion four years later. KRAS variants have been shown to be present in low grade ovarian cancer and well-differentiated tumors.

Conclusion* Borderline tumors often recurs in form of borderline tumor but the genetic profile should not be the same as the primary tumor, as shown in our case report. Loss of KRAS could explain the recurrence of the disease. Seeing that the molecular profile of the tumor is in constant change, a continuity in the spectrum normal-benign-borderline-malignant could be hypothesized.

\section{PREVENTION OF INCISIONAL HERNIA IN OVARIAN CANCER PATIENTS UNDERGOING MIDLINE LAPAROTOMY}

${ }^{1}$ B Fabrego Capdevila*, ${ }^{1} E$ Miralpeix, ${ }^{1} \mathrm{JM}$ Sole-Sedeño, ${ }^{2} \mathrm{~J}$ A Pereira-Rodriguez, ${ }^{1} \mathrm{G}$ Mancebo. ${ }^{1}$ Hospital del Mar, Department of Obstetrics and Gynecology, Barcelona, Spain; ${ }^{2}$ Hospital del Mar, Department of Surgery, Barcelona, Spain

\subsection{6/ijgc-2021-ESGO.405}

Introduction/Background* Incisional hernias (IH) are a frequent complication of midline laparotomies in abdominal surgery. This study was conducted in order to determine the efficacy of mesh placement and assess the optimal fascia suture technique to reduce the $\mathrm{IH}$ rate in patients surgically treated after being diagnosed with malignant or borderline ovarian tumors.

Methodology Retrospective data from patients undergoing midline laparotomy for borderline or ovarian cancer in Hospital del Mar, Barcelona, from January 2008 to December 2019 were collected. Patient demographic, preoperative and intraoperative characteristics and potential risk factors for hernia were reported. The incidence of $\mathrm{IH}$ between groups (mesh and non-mesh) and the technique used in fascial closure for each patient (small bites technique vs large tissue bites) was reported.

Result(s)* In total, 133 patients with available data for followup were included. After clinical and radiological examination, $25(18.79 \%)$ of them showed IH. 18 of $61(29.5 \%)$ patients in non-mesh group developed $\mathrm{IH}$, compared with 7 of 72 (9.7\%) in mesh group (OR 0.25, 95\% CI 0.09-0.66, $\mathrm{p}<0.005)$. Patients of large tissue bites group showed higher prevalence of $\mathrm{IH}$ compared with small bites technique group without statistical significance (OR 0.46, 95\% CI 0.17-1.24, $\mathrm{p}=0.119)$. The combination of mesh reinforcement and small bites technique for fascial closure significantly reduce IH risk $(\mathrm{p}=0.021)$.

Conclusion* The incidence of IH is high in patients undergoing midline laparotomy for ovarian cancer or borderline ovarian tumor. The addition of a prophylactic mesh and the use of small bites technique may reduce the incidence of $\mathrm{IH}$ and potentially minimize the social impact and costs of this complication.

\section{MARKERS ON PLATELET MICROVESICLES FOR DIAGNOSTICS OF OVARIAN CANCER}

${ }^{1}$ E Karlsson, ${ }^{2} \mathrm{~F}$ Mobarrez, ${ }^{3} \mathrm{E}$ Epstein, ${ }^{4} \mathrm{G}$ Auer, ${ }^{5} \mathrm{~J}$ Widengren, ${ }^{1} \mathrm{M}$ Lomnytska*. ${ }^{1}$ Uppsala University, Women's and Children's health, Uppsala, Sweden; '2uppsala University, Department of Medical Sciences, Clinical Chemistry, Uppsala, Sweden; ${ }^{3}$ Karolinska Institute, Women's and Children's health, Stockholm, Sweden; ${ }^{4}$ Karolinska Institute, Oncology and Pathology, Stockholm, Sweden; ${ }^{5}$ Royal Technical University, Stockholm, Sweden

\subsection{6/ijgc-2021-ESGO.406}

Introduction/Background* Routine blood markers provide poor diagnostic capacity for ovarian cancer. Ultrasound examination using the criteria developed by International Ovarian Tumor Analysis group is the most sensitive and specific diagnostic method, but in up to $20 \%$ of cases evaluation is inconclusive (Sladkevicius et al. 2020; Valentin et al. 2011). We pioneered platelet proteome analysis and identified platelet biomarkers of ovarian cancer (Lomnytska et al. 2018).

Methodology The purpose of the study is to identify diagnostic marker panel on platelet microvesicles in blood plasma for non-invasive differential diagnostics of benign adnexal lesions, borderline tumours and ovarian cancer. The expression of platelet protein biomarkers on platelet microvesicles in patients with benign and malignant adnexal lesions was analysed using flow cytometry. Identified biomarker panels were analysed together with the gynaecologic ultrasound criteria.

Result(s)* Analysis comprised 39 patients with benign adnexal lesions $(n=10)$, borderline $(n=10)$, ovarian cancer stage I-II $(\mathrm{n}=8)$ and stage III-IV $(\mathrm{n}=11)$. Using flow cytometry analysis of platelet microvesicles in platelet-poor blood plasma, we detected our previously identified by proteome analysis of platelets markers ACTN4, CRKL, ERP29, GELS, PHB and SRC

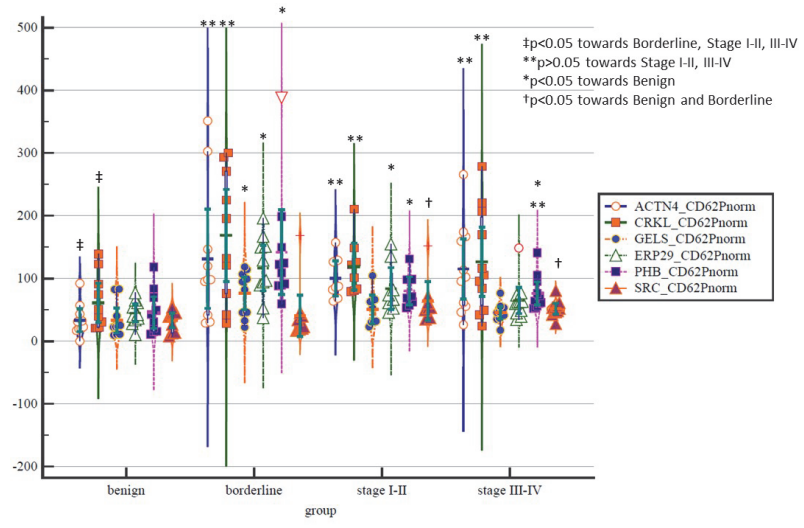

Abstract 464 Figure 1 
(Lomnytska et al., 2018). Expression of P-selectin positive platelet microvesicles was 2-fold higher in ovarian cancer $(\mathrm{p}<0.05)$. Increased expression of our identified markers on P-selectin positive platelet microvesicles was observed in ovarian cancer (figure 1).

Conclusion* Identified platelet biomarkers (Lomnytska et al. 2018) are detectable on platelet microvesicles in blood plasma with increased expression in ovarian cancer. Further analysis of microvesicles in relation to ultrasound evaluation has the potential to improve ovarian cancer diagnostics.

\section{ULTRASTAGING PROTOCOL IN SENTINEL LYMPH NODE FOR APPARENT EARLY STAGE OVARIAN CANCER}

V Lago*, B Montero, S Lopez, P Padilla Iserte, MT Luis Javier, B Segarra Vidal, S Domingo. University Hospital La Fe, Spain

\subsection{6/ijgc-2021-ESG0.407}

Introduction/Background* The objective of the present study is to determine the role of sentinel lymph node (SLN) ultrastaging in apparent early-stage ovarian cancer.

Methodology We previously demonstrated the feasibility of SLN in early-stage ovarian cancer in a pilot study and in a clinical trial (NCT03452982). The SLN of the 30 patients involved in both were processed following an ultrastaging protocol. The cost of ultrastaging processing was also reported.

Result(s)* A SLN was detected in up to $91.3 \%$ and $90 \%$ in the pelvic and para-aortic region, respectively. In all cases, a SLN was detected at least in one field, pelvic or para-aortic. The mean time from injection to SLN resection was $53.3 \pm$ $20.3 \mathrm{~min}$. Two of $30(6.6 \%)$ patients had a contralateral SLN in the para-aortic field, but no patients had contralateral SLN within the pelvic field after injection. The mean number of harvested SLN was $2.1 \pm 1.4$ (range: 0-5) and $2.7 \pm 1.5$ (range: $0-7$ ) in the pelvic and para-aortic region, respectively. Two patients were upgraded to stage IIIA1 because of lymph node metastasis. In the first case, based on single sections and haematoxylin and eosin (H\&E) examination, a pelvic
SLN micrometastasis $(1 \mathrm{~mm})$ was found on the first $\mathrm{H} \& \mathrm{E}$ section. By using the ultrastaging protocol, the size of the metastasis was increased to $2.1 \mathrm{~mm}$ (macrometastasis). In the same patient, the ultrastaging study of the inframesenteric para-cava SLNs found isolated tumour cells in the subcapsular and interfollicular lymph nodes sinus in one of the two SLN harvested (in one of the sections at the fourth and fifth ultrastage levels). The other upstaged case was a para-aortic macrometastasis in a patient in whom the SLN was not identified in the para-aortic field because of the absence of migration from the infundibulo-pelvic stump injection. The cost of ultrastaging in on average $96.8 €$ (range: 0-230.5) and $124.5 €$ (range: 0-322.7€) for pelvic and para-aortic SLN, respectively.

Conclusion* A uniform protocol for ultrastaging is essential for lower-volume metastasis detection and to provide reproducible information between upcoming studies, as evidence about SLN in ovarian cancer is growing.

\section{Ovarian cancer sentinel lymph node ultrastaging protocol}

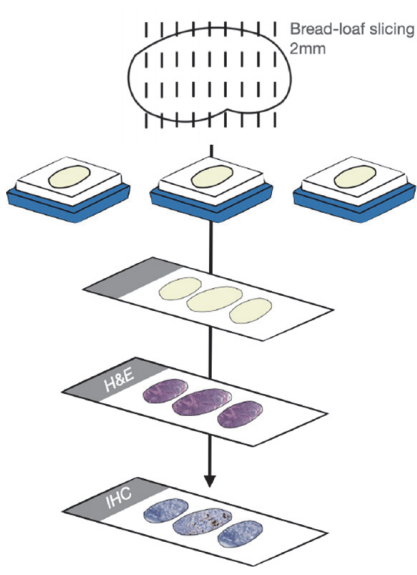

Macroscopic study Sections of approximately
$2 \mathrm{~mm}$ perpendicular to the largest diameter of the node

Processing and microscopic study Serial study was carried out with levels deepening every 200 microns (with a limit of 6 levels at most or until exhausted)

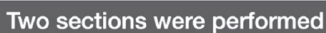
at each level:

1st with $\mathrm{H} \& \mathrm{E}$

If negative: IHC CK AE1/3

Abstract 468 Figure 2
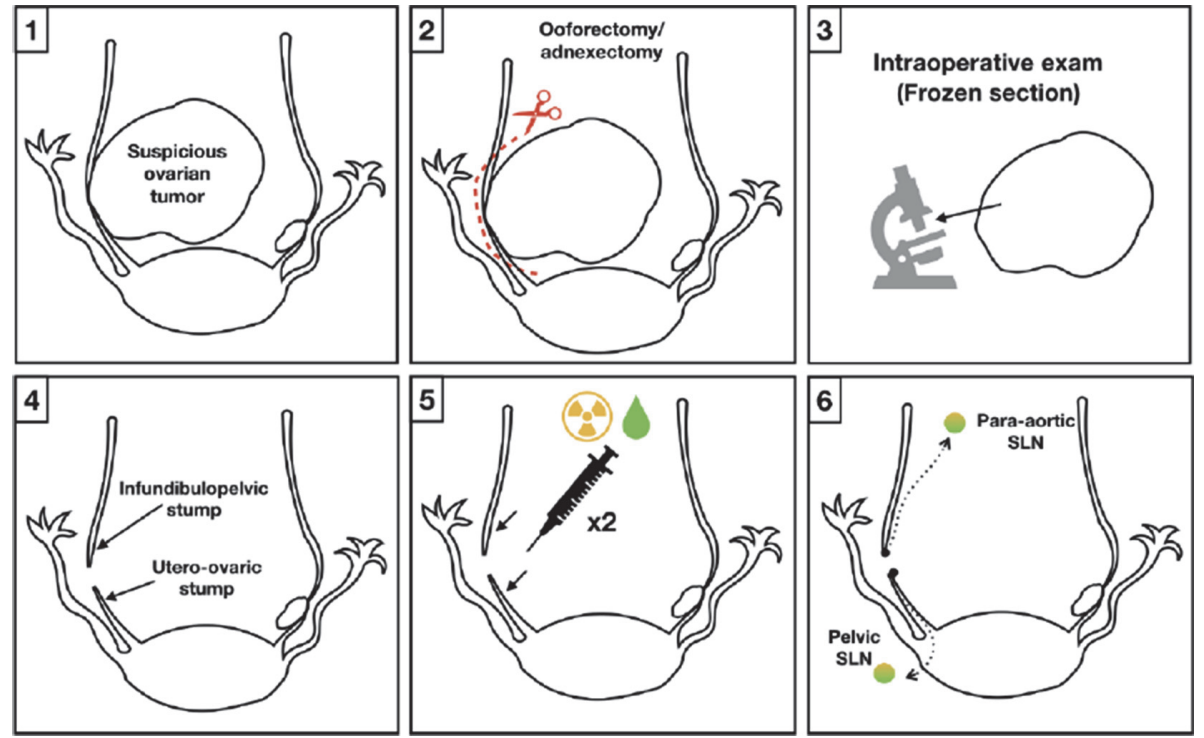

Abstract 468 Figure 1 